\title{
Theory of Disclinations: III. Continuous and Discrete Disclinations in Isotropic Elasticity
}

\author{
Roland deWit \\ Institute for Materials Research, National Bureau of Standards, Washington, D.C. 20234
}

(February 22, 1973)

\begin{abstract}
A general theory of stationary disclinations is developed for a linearly elastic, infinitely extended, homogeneous, isotropic body. It is shown to be a special case of the anisotropic theory. Integral expressions are derived for the total displacement, elastic strain and bend-twist, and stress. Both continuous distributions and discrete lines are treated.
\end{abstract}

Key words: Burgers vector; continuum mechanics; defect; disclination; dislocation; distortion; Green's tensor; incompatibility; plasticity; strain.

1. Introduction ............................... 359

2. Isotropy .................................. 360

3. Review of incompatible theory ............. 360

3.1. The plastic strain problem............ 360

3.2. Application: The point defect.......... 361

3.3. The incompatibility problem........... 362

4. Review of dislocation theory

\section{Contents}

5. Continuous distribution of defects........... 364

6. The discrete defect line ................... 366

7. Summary ............................... 367

362 8. References............................... 368

\section{Introduction}

In a previous paper [2] ${ }^{1}$ we have developed the general theory of stationary disclinations in a linearly elastic, infinitely extended, homogeneous, anisotropic body. In the present paper we wish to specialize some of those results to the case of an isotropic material.

The purpose for this is three-fold. First, for many special applications of the general theory, the isotropic calculations can be carried through analytically; therefore it is convenient to have the isotropic formulas. Second, it will be easier to compare the present results with those in the general lietature; many of the latter have been derived independently with isotropy assumed a priori. Third, when the relation between isotropic and anisotropic theory is clearly revealed, it is often possible to adapt isotropic techniques to anisotropic problems; this has often been

\footnotetext{
${ }^{1}$ Figures in brackets indic ate the literature references at the end of this paper.
}

successful, and the literature on isotropy is vastly greater than that on anisotropy.

The general approach in this paper is to develop the isotropic theory $a b$ initio and show its correspondence to the anisotropic results at many convenient and illustrative points.

In section 2 we give the isotropic forms of certain key relations, i.e., the elastic constants, Green's tensor, and the incompatibility source tensor.

In section 3 we review incompatible elasticity theory without specifying the nature of the defects. We first solve the plastic strain problem which gives the integral expression for the total displacement, which is useful for all the rest of defect theory. We illustrate the result by Eshelby's simplest model of a point defect. Second, we solve the incompatibility problem, which gives the state quantity of elastic strain as an integral over the incompatibility tensor. 
In section 4 we review classical dislocation theory. In section 5 we develop the theory of a continuous distribution of defects. Integral expressions are derived for the basic elastic fields (i.e., strain and bend-twist) and the stress. The expressions for these state quantities are given in terms of the defect densities, i.e., the dislocation and disclination densities.

In section 6 we specialize to the discrete defect line. We give the total displacement as a surface integral, and the basic elastic fields and stress as line integrals.

In this paper we shall freely refer to the results of two previous papers [1,2], which will be denoted as I and II.

\section{Isotropy}

For an isotropic material the elastic constants $C_{i j k l}$ reduce to only two independent constants. In terms of the modulus of rigidity $G$ and Poisson's ratio $\nu$ we can express them as follows [3]:

$$
C_{i j k l}=2 G\left(\delta_{i k} \delta_{j l}+\frac{\nu}{1-2 \nu} \delta_{i j} \delta_{k l}\right)_{(i j)},
$$

where $\delta_{i j}$ is the Kronecker delta, and the symbol $(i j)$ indicates that the quantity in parentheses is symmetrized with respect to $i$ and $j$. We have used Cartesian coordinates for simplicity.

For isotropy it is also possible to give the explicit expression of Green's tensor, as follows [3]:

$$
G_{j n}(\mathbf{r})=\frac{1}{8 \pi G}\left(\delta_{j n} r_{, q q}-\frac{1}{2(1-\nu)} r_{, j n}\right),
$$

where $r$ is the radius vector, and the comma indicates differentiation. The combination $C_{i j k l} G_{j n, i}$ occurs extensively throughout II. From (2.1-2) we find that it reduces to

$$
\begin{aligned}
C_{i j k l} G_{j n, i}(\mathbf{r})=\frac{1}{8 \pi} & \left(\delta_{l n} r_{, k q q}+\delta_{k n} r_{, l q q}\right. \\
& \left.-\frac{1}{1-\nu} r_{, k l n}+\frac{\nu}{1-\nu} \delta_{k l, n q q}\right) .
\end{aligned}
$$

Another quantity that occurs in II is the incompatibility source tensor, defined by (II4.25) ${ }^{2}$

$I_{m n p q}(\mathbf{r})=\frac{1}{4 \pi} \int \epsilon_{p m k} \epsilon_{q s l} C_{i j k l} G_{j n, i^{\prime} s^{\prime}}\left(\mathbf{r}^{\prime}\right) R^{-1} d V^{\prime}{ }_{(m n)}$,

where $\epsilon_{p m k}$ is the permutation symbol and $R$ is the magnitude of the vector $\mathbf{R}=\mathbf{r}-\mathbf{r}^{\prime}$. From (2.3) this reduces for isotropy to

$$
\begin{aligned}
I_{m n p q}(\mathbf{r})= & \frac{1}{32 \pi^{2}} \int \epsilon_{p m k} \epsilon_{q s l}\left(\delta_{l n} r^{\prime},{ }_{i^{\prime} i^{\prime} k^{\prime} s^{\prime}}\right. \\
& \left.+\frac{\nu}{1-\nu} \delta_{k l} r^{\prime},{ }_{i^{\prime} i^{\prime} n^{\prime} s^{\prime}}\right) R^{-1} d V^{\prime}{ }_{(m n)},
\end{aligned}
$$

where we have used the antisymmetry or skewsymmetry of $\epsilon_{q s l}$. From the integral

$$
\int r^{\prime},{ }_{i^{\prime} i^{\prime}} R^{-1} d V^{\prime}=2 \int\left(r^{\prime} R\right)^{-1} d V^{\prime}=-4 \pi r
$$

this reduces by partial integration to

$$
I_{m n p q}(\mathbf{r})=-\frac{1}{8 \pi} \epsilon_{p m k} \epsilon_{q s l}\left(\delta_{l n} r_{, k s}+\frac{\nu}{1-\nu} \delta_{k l} r_{, n s}\right)_{(m n)} .
$$

Finally by (II4.26) this reduces to

$$
\begin{gathered}
I_{m n p q}(\mathbf{r})=-\frac{1}{8 \pi}\left[\delta_{m q} \delta_{n p} r_{, k k}-\delta_{m n} \delta_{p q} r_{, k k}+\delta_{m n} r_{, p q}\right. \\
-\delta_{n p} r, m q \\
\left.+\frac{1}{1-\nu}\left(\delta_{p q} r_{, m n}-\delta_{m q} r_{, n p}\right)\right]_{(m n)}
\end{gathered}
$$

The specializations of this section will reduce all the results of II to isotropy, as we shall see. Throughout the paper repeated indices are summed according to the Einstein summation convention. This convention also applies between primed and unprimed indices, as in (2.4).

\section{Review of Incompatible Theory}

In this section we shall give the formal solution of two problems, which can be posed without specifying the nature of the defects involved, i.e., the plastic strain problem and the incompatibility problem.

\subsection{The Plastic Strain Problem}

Given an infinitely extended homogeneous isotropic body with the plastic strain $e_{i j}^{P}$ given as a prescribed function of space. We seek to find the resulting displacement $u_{i}^{T}$ throughout the body.

We solve this problem by a method similar to that of section II2. ${ }^{3}$ The equilibrium equation for the stress $\sigma_{i j}$ is (II2.1)

$$
\sigma_{i j, i}=0 .
$$

For the isotropic case Hooke's law (II2.2) reduces by (2.1) to

$$
\sigma_{i j}=2 G\left(e_{i j}+\frac{\nu}{1-2 \nu} \delta_{i j} e\right)
$$

where we have written $e$ for dilatation, or trace of the elastic strain $e_{i j}$, i.e., $e=e_{i i}$. The total strain is defined in terms of the total displacement (II2.4) and consists of an elastic and a plastic part (II2.5)

$$
e_{i j}^{T} \equiv u_{(j, i)}^{T}=e_{i j}+e_{i j}^{P} .
$$

The above relations $(3.1-3)$ are conveniently combined into the expression (c.f. II2.6)

${ }^{3}$ Section II2 is Section 2 in ref. [2] 


$$
u_{i, j j}^{T}+\frac{1}{1-2 \nu} u_{j, i j}^{T}=2 e_{i j, j}^{P}+\frac{2 \nu}{1-2 \nu} e_{, i}^{P}
$$

This is the set of partial differential equations we have to solve for $u_{i}^{T}$ when $e_{i j}^{P}$ is given. The most straightforward method of attack is to reduce (3.4) to a biharmonic equation. First we take the derivative of (3.4) with respect to $x_{i}$ to obtain

$$
\frac{1-\nu}{1-2 \nu} u_{i, i j j}^{T}=e_{i j, i j}^{P}+\frac{\nu}{1-2 \nu} e_{, i i}^{P} .
$$

Next, we take the Laplacian operator of (3.4) and find

$$
u_{i, j j k k}^{T}+\frac{1}{1-2 \nu} u_{j, i j k k}^{T}=2 e_{i j, j k k}^{P}+\frac{2 \nu}{1-2 \nu} e_{, i j j}^{P} .
$$

Combining (3.5-6) we have

$u_{i, j j k k}^{T}=2 e_{i j, j k k}^{P}-\frac{1}{1-\nu} e_{j k, i j k}^{P}+\frac{\nu}{1-\nu} e_{, i j j}^{P}$.

To integrate this equation we shall use the following relation

$$
(8 \pi)^{-1} r_{, j j k k}+\delta(\mathbf{r})=0,
$$

where $\delta(\mathbf{r})$ is the three-dimensional Dirac delta function, defined in Appendix IIB. One interesting way to derive this relation is by substituting (2.3) into (II2.7). This shows that (3.8) is the isotropic equivalent of the defining equation for Green's function.

The solution of our problem now follows easily

$$
\begin{aligned}
u_{i}^{T}(\mathbf{r})= & \int \delta(\mathbf{R}) u_{i}^{T}\left(\mathbf{r}^{\prime}\right) d V^{\prime} \\
= & -\frac{1}{8 \pi} \int R, j j k k u_{i}^{T}\left(\mathbf{r}^{\prime}\right) d V^{\prime} \\
= & -\frac{1}{8 \pi} \int R u_{i, j^{\prime} j^{\prime} k^{\prime} k^{\prime}}^{T}\left(\mathbf{r}^{\prime}\right) d V^{\prime} \\
= & -\frac{1}{8 \pi} \int R\left[2 e_{i j, j^{\prime} k^{\prime} k^{\prime}}^{P}\left(\mathbf{r}^{\prime}\right)-\frac{1}{1-\nu} e_{j k, i^{\prime} j^{\prime} k^{\prime}}^{P}\left(\mathbf{r}^{\prime}\right)\right. \\
& \left.+\frac{\nu}{1-\nu} e_{, i^{\prime} j^{\prime} j^{\prime}}^{P}\left(\mathbf{r}^{\prime}\right)\right] d V^{\prime} \\
= & \frac{1}{8 \pi} \int\left[2 R_{, j k k} e_{i j}^{P}\left(\mathbf{r}^{\prime}\right)-\frac{1}{1-\nu} R_{, i j k} e_{j k}^{P}\left(\mathbf{r}^{\prime}\right)\right. \\
& \left.+\frac{\nu}{1-\nu} R_{, i j j} e^{P}\left(\mathbf{r}^{\prime}\right)\right] d V^{\prime},
\end{aligned}
$$

where the integrations are taken over all space. In the above derivation the first equality follows from the property of the delta function (IIB3), the second equality from (3.8), the third by partial integrations, the fourth from (3.7), and the fifth again by partial integrations. In the above partial integrations the surface integrals at infinity vanish. For this to happen the following two conditions must be satisfied: the boundary condition for the total displacement is that $u_{i}^{T}(\mathbf{r}) \rightarrow 0$ as $r \rightarrow \infty$; and the condition on the prescribed plastic strain is that $e_{i j}^{P}(\mathbf{r})$ approaches zero faster than $r^{-1}$ as $r \rightarrow \infty$. This last condition also insures that the integral (3.9) is finite. It is easily shown that (3.9) also follows by substituting (2.3) into (II2.15), verifying the consistency between isotropic and anisotropic theory. For the case that $e_{i j}^{P}$ is constant within a bounded volume and vanishes outside this volume, (3.9) immediately reduces to Eshelby's result [4]. The total distortion is easily obtained from (3.9)

$$
\begin{aligned}
\beta_{i j}^{T}(\mathbf{r})= & u_{j, i}^{T}(\mathbf{r}) \\
= & -\frac{1}{8 \pi} \int\left[2 R_{, i k l l} e_{j k}^{P}\left(\mathbf{r}^{\prime}\right)-\frac{1}{1-\nu} R_{, i j k l} e_{k l}^{P}\left(\mathbf{r}^{\prime}\right)\right. \\
& \left.+\frac{\nu}{1-\nu} R_{, i j k k} e^{P}\left(\mathbf{r}^{\prime}\right)\right] d V^{\prime}
\end{aligned}
$$

The equations of this section will form the basis of the expressions for the fields of the more specific defects treated in subsequent sections.

Eshelby pointed out that the field of a defect can also be simulated by a fictitious distribution of body force. For the isotropic case it is obtained by substituting (2.1) into (II2.16). So the defect described by $e_{i j}^{P}$ is simulated by the fictitious body force

$$
-f_{j}=-2 G\left(e_{i j, i}^{P}+\frac{\nu}{1-2 \nu} e_{, j}^{P}\right) .
$$

\subsection{Application: The Point Defect}

It will be instructive to consider a simple application of the relations (3.9) and (3.11). Eshelby [5] regards the discrete point dilatation as the simplest elastic model of a point defect. In our formulation this is easily given by the expression

$$
e_{i j}^{P}(\mathbf{r})=\frac{1}{3} \Delta V \delta_{i j} \delta(\mathbf{r}),
$$

where $\Delta V$ is a constant which represents the plastic volume change. Then we find for (3.9)

$$
u_{i}^{T}(\mathbf{r})=c x_{i} / r^{3},
$$

where $c$ is the "strength" of the point defect:

$$
c=\frac{1+\nu}{1-\nu} \frac{\Delta V}{12 \pi}
$$


From (3.11) we find that this point defect can be simulated by the fictitious body force

$$
-f_{j}=-\Delta V K \delta_{, j}(\mathbf{r}),
$$

where $K$ is the bulk modulus

$$
K=\frac{1+\nu}{1-2 \nu} \frac{2 G}{3}
$$

These results agree with Eshelby, who elaborates this problem further.

In view of section II3.3 the plastic distortion $\beta_{i j}^{P}$ can be regarded as equivalent to a dislocation loop density $\gamma_{i j}$ (II3.34). Hence eq (3.12) shows that the point defect can be represented by a set of three equal discrete prismatic loops at right angles. This then is an example showing that a point defect can be represented by a discrete defect loop density.

Equation (3.15) furthermore shows that the point defect can also be simulated by a set of three equal fictitious discrete double forces at right angles.

\subsection{The Incompatibility Problem}

The incompatibility tensor $\eta_{i j}$ is given as a prescribed function of space. To find the resulting elastic strain $e_{i j}$ throughout the body.

We solve this problem by exploiting the concept of plastic strain. If the plastic strain field $e_{i j}^{P}$ is given, then the symmetric incompatibility tensor is defined by (II4.11)

$$
\eta_{i j} \equiv \epsilon_{i k l} \epsilon_{j m n} \epsilon_{l n, k m}^{P}
$$

and satisfies the continuity condition (II4.13)

$$
\eta_{i j, i}=0 \text {. }
$$

The expression (3.17) can be expanded by (II4.26) into $\eta_{i j}=-e_{i j, k k}^{P}-e_{, i j}^{P}+e_{j k, i k}^{P}+e_{i k, j k}^{P}+\delta_{i j}\left(e_{, l l}^{P}-e_{k l, k l}^{P}\right)$.

From this we derive the two results

$$
\begin{gathered}
\eta=e_{, l l}^{P}-e_{k l, k l}^{P}, \\
\eta_{i j}-\delta_{i j} \eta=-e_{i j, k k}^{P}-e_{, i j}^{P}+e_{j k, i k}^{P}+e_{i k, j k}^{P} .
\end{gathered}
$$

The solution of our problem now follows easily:

$$
\begin{aligned}
e_{i j}(\mathbf{r})= & -\frac{1}{8 \pi} \int\left[R_{, l l}\left(e_{j k, i^{\prime} k^{\prime}}^{P}+e_{i k, j^{\prime} k^{\prime}}^{P}\right)\right. \\
& \left.-\frac{1}{1-\nu} R_{, i j} e_{k l, k^{\prime} l^{\prime}}^{P}+\frac{\nu}{1-\nu} R_{, k k} e_{, i^{\prime} j^{\prime}}^{P}\right] d V^{\prime} \\
& -e_{i j}^{P}(\mathbf{r}) \\
= & -\frac{1}{8 \pi} \int\left[R_{, l l}\left(\eta_{i j}-\delta_{i j} \eta+e_{i j, k^{\prime} k^{\prime}}^{P}+e_{, i^{\prime} j^{\prime}}^{P}\right)\right.
\end{aligned}
$$

$$
\begin{aligned}
& \left.+\frac{1}{1-\nu} R_{, i j}\left(\eta-e_{, l^{\prime} l^{\prime}}^{P}\right)+\frac{\nu}{1-\nu} R_{, k k} e_{, i^{\prime} j^{\prime}}^{P}\right] d V^{\prime} \\
& -e_{i j}^{P}(\mathbf{r}) \\
& =-\frac{1}{8 \pi} \int\left[R_{, k k} \eta_{i j}\left(\mathbf{r}^{\prime}\right)\right. \\
& \left.+\left(\frac{R, i j}{1-\nu}-\delta_{i j} R_{, k k}\right) \eta\left(\mathbf{r}^{\prime}\right)\right] d V^{\prime}
\end{aligned}
$$

Here the first equality follows from (3.3), (3.10), and some partial integrations, the second equality from (3.20-21), and the third by partial integrations, (3.8), and cancellations. It is easily shown that (3.22) also follows by substituting (2.8) into (II4.31), and using (3.18). In the latter calculation it will be noted that the terms in (2.8) containing $r_{, p}$ and $r_{, q}$ make no contribution to the integral. This shows that the precise form of (2.8) is somewhat indeterminate.

We see that the elastic strain $e_{i j}$ is a state quantity, because it is given entirely in terms of an integral over the incompatibility $\eta_{i j}$, which represents a defect distribution, and is therefore itself also a state quantity. By a state quantity we mean a quantity that can be measured experimentally without any knowledge of former states of the body.

\section{Review of Dislocation Theory}

\subsection{Continuous Distribution of Dislocations}

In this section we give a formal solution to the following problem: The plastic distortion $\beta_{i j}^{P}$, or the dislocation density $\alpha_{i n}$, is given as a prescribed function of space. To find the resulting elastic distortion $\beta_{i j}$.

We solve this problem basically by using the solution for the displacement found in section 3.1. We also derive expressions for the strain, dilatation, stress, and rotation. The dislocation density is defined by (II3.4)

$$
\alpha_{i n} \equiv-\epsilon_{i k l} \beta_{l n, k}^{P}
$$

and satisifies the continuity condition (II3.6)

$$
\alpha_{i n, i}=0 .
$$

Instead of the dislocation density it is sometimes convenient to use the contortion (I5.11b)

$$
K_{l k}=\frac{1}{2} \delta_{k l} \alpha-\alpha_{k l} .
$$

The total distortion is defined in terms of the total displacement and for dislocations only consists of an elastic and plastic part (II3.9)

$$
\beta_{i j}^{T} \equiv u_{j, i}^{T}=\beta_{i j}+\beta_{i j}^{P} .
$$


Once the elastic distortion is found, the elastic strain and rotation easily follow from (II3.13-14)

$$
\begin{aligned}
& e_{i j}=\beta_{(i j)}, \\
& \omega_{k}=\frac{1}{2} \epsilon_{i j k} \beta_{i j} .
\end{aligned}
$$

To solve our problem we recall that the plastic strain is the symmetric part of the plastic distortion (II3.2)

$$
e_{i j}^{P}=\beta_{(i j)}^{P},
$$

and therefore we find the total displacement in terms of the plastic distortion from (3.9)

$$
\begin{aligned}
& u_{i}^{T}(\mathbf{r})=-\frac{1}{8 \pi} \int\left[R_{, j j k} \beta_{i k}^{P}\left(\mathbf{r}^{\prime}\right)+R, j k k \beta_{j i}^{P}\left(\mathbf{r}^{\prime}\right)\right. \\
& \left.-\frac{1}{1-\nu} R_{, i j k} \beta_{j k}^{P}\left(\mathbf{r}^{\prime}\right)+\frac{\nu}{1-\nu} R_{, i j j} \beta^{P}\left(\mathbf{r}^{\prime}\right)\right] d V^{\prime} .
\end{aligned}
$$

By (4.4) it is then simple to express the elastic distortion in terms of the plastic distortion, which solves the first part of our problem. Next, we want to find the expression in terms of the dislocation density:

$$
\begin{aligned}
\beta_{i j}(\mathbf{r})= & -\frac{1}{8 \pi} \int\left[R,{ }_{l n n} \beta_{j l, i^{\prime}}^{P}+R_{, k n n} \beta_{k j, i^{\prime}}^{P}\right. \\
& \left.-\frac{1}{1-\nu} R_{, j k l} \beta_{k l, i^{\prime}}^{P}+\frac{\nu}{1-\nu} R_{, j n n} \beta_{, i^{\prime}}^{P}\right] d V^{\prime}-\beta_{i j}^{P}(\mathbf{r}) \\
= & \frac{1}{8 \pi} \int\left[R,{ }_{l n n}\left(\epsilon_{i j k} \alpha_{k l}-\beta_{i l, j^{\prime}}^{P}\right)\right. \\
& +R_{, k n n}\left(\epsilon_{i k l} \alpha_{l j}-\beta_{i j, k^{\prime}}^{P}\right) \\
& -\frac{1}{1-\nu} R_{, j k l}\left(\epsilon_{i k m} \alpha_{m l}-\beta_{i l, k^{\prime}}^{P}\right) \\
& \left.+\frac{\nu}{1-\nu} R_{, j n n}\left(\epsilon_{i l m} \alpha_{m l}-\beta_{i l, l^{\prime}}^{P}\right)\right] d V^{\prime}-\beta_{i j}^{P}(\mathbf{r}) \\
= & \frac{1}{8 \pi}\left[R_{, l n n} \epsilon_{i j k} \alpha_{k l}\left(\mathbf{r}^{\prime}\right)+R_{, k n n} \epsilon_{i k l} \alpha_{l j}(\mathbf{r})\right. \\
& -\frac{1}{1-\nu} R_{, j k l} \epsilon_{i k m} \alpha_{m l}\left(\mathbf{r}^{\prime}\right) \\
& \left.+\frac{\nu}{1-\nu} R_{, j n n} \epsilon_{i l m} \alpha_{m l}\left(\mathbf{r}^{\prime}\right)\right] d V^{\prime} .
\end{aligned}
$$

Here the first equality follows from (4.4), (4.8) and some partial integrations, the second from (4.1), and the third by partial integrations, (3.8), and some cancellations. This result could also have been obtained more directly by substituting (2.3) into (II3.17).
There is another convenient form for (4.9), which is obtained by using the identities

$$
\begin{gathered}
\epsilon_{i k l} \alpha_{l j} \equiv \epsilon_{i k l} \alpha_{j l}-\epsilon_{k l m} \delta_{i j} \alpha_{m l}+\epsilon_{i l m} \delta_{j k} \alpha_{m l} \\
R_{, l} \epsilon_{i k m} \equiv R, m \epsilon_{i k l}-R_{, i} \epsilon_{k l m}+R_{, k} \epsilon_{i l m}
\end{gathered}
$$

With these we find

$$
\begin{gathered}
\beta_{i j}(\mathbf{r})=\frac{1}{8 \pi} \int\left[R_{, l n n} \epsilon_{i j k} \alpha_{k l}\left(\mathbf{r}^{\prime}\right)+R_{, k n n} \epsilon_{i k l} \alpha_{j l}\left(\mathbf{r}^{\prime}\right)\right. \\
-R_{, k n n} \epsilon_{k l m} \delta_{i j} \alpha_{m l}\left(\mathbf{r}^{\prime}\right) \\
\left.+\frac{1}{1-\nu}\left(R_{, i j k} \epsilon_{k l m}-R_{, j k m} \epsilon_{i k l}\right) \alpha_{m l}\left(\mathbf{r}^{\prime}\right)\right] d V^{\prime}
\end{gathered}
$$

The last term in this expression vanishes by a partial integration and (4.2). So we obtain finally

$$
\begin{aligned}
\beta_{i j}(\mathbf{r})= & \frac{1}{8 \pi} \int\left[R_{, l n n} \epsilon_{i j k} \alpha_{k l}\left(\mathbf{r}^{\prime}\right)+R_{, k n n} \epsilon_{i k l} \alpha_{j l}\left(\mathbf{r}^{\prime}\right)\right. \\
& \left.+\left(\frac{R_{, i j k}}{1-\nu}-\delta_{i j} R_{, k n n}\right) \epsilon_{k l m} \alpha_{m l}\left(\mathbf{r}^{\prime}\right)\right] d V^{\prime}
\end{aligned}
$$

This is the elastic distortion due to the prescribed dislocation distribution $\alpha_{i n}$. It is a state quantity. By (4.1) we can also express it in terms of the plastic distortion. This then solves the originally posed problem.

Next, we find the elastic strain from (4.5) and (4.13)

$$
\begin{aligned}
e_{i j}(\mathbf{r}) & =\frac{1}{8 \pi} \int\left[R_{, k n n}\left(\epsilon_{i k l} \alpha_{j l}\left(\mathbf{r}^{\prime}\right)\right)_{(i j)}\right. \\
& \left.+\left(\frac{R_{, i j k}}{1-\nu}-\delta_{i j} R_{, k n n}\right) \epsilon_{k l m} \alpha_{m l}\left(\mathbf{r}^{\prime}\right)\right] d V^{\prime} .
\end{aligned}
$$

From this we find the dilatation

$$
e(\mathbf{r})=-\frac{1-2 \nu}{1-\nu} \frac{1}{8 \pi} \int R_{, k n n} \epsilon_{k l m} \alpha_{m l}\left(\mathbf{r}^{\prime}\right) d V^{\prime},
$$

and the stress by (3.2)

$$
\begin{aligned}
& \sigma_{i j}(\mathbf{r})=\frac{G}{4 \pi} \int\left[R_{, k n n}\left(\epsilon_{i k l} \alpha_{j l}\left(\mathbf{r}^{\prime}\right)\right)_{(i j)}\right. \\
& \left.+\frac{1}{1-\nu}\left(R_{, i j k}-\delta_{i j} R_{, k n n}\right) \epsilon_{k l m} \alpha_{m l}\left(\mathbf{r}^{\prime}\right)\right] d V^{\prime} .
\end{aligned}
$$

The rotation is found from (4.6) and (4.13)

$$
\begin{array}{r}
\omega_{k}(\mathbf{r})=\frac{1}{8 \pi} \int\left[R_{, l n n} \alpha_{k l}\left(\mathbf{r}^{\prime}\right)-\frac{1}{2} R_{, k n n} \alpha\left(\mathbf{r}^{\prime}\right)\right. \\
\left.+\frac{1}{2} R_{, j n n} \alpha_{j k}\left(\mathbf{r}^{\prime}\right)\right] d V^{\prime} .
\end{array}
$$


The last term in this expression vanishes by a partial integration and (4.2). So we have

$$
\begin{aligned}
\omega_{k}(\mathbf{r}) & =\frac{1}{8 \pi} \int\left[R_{, l n n} \alpha_{k l}\left(\mathbf{r}^{\prime}\right)-\frac{1}{2} R_{, k n n} \alpha\left(\mathrm{r}^{\prime}\right)\right] d V^{\prime} \\
& =-\frac{1}{8 \pi} \int R_{, l n n} K_{l k}\left(\mathbf{r}^{\prime}\right) d V^{\prime}
\end{aligned}
$$

where the last equality follows from (4.3).

To summarize, in this section we have derived explicit integral expressions for the most useful elastic fields (distortion, strain, dilatation, stress, and rotation) of a continuous distribution of dislocations in an infinite isotropic body.

\subsection{The Discrete Dislocation Line}

For a discrete dislocation line with Burgers vector $b_{j}$ along the curve $L$ the plastic distortion has the special form (II3.21)

$$
\beta_{i j}^{P}(\mathbf{r})=-\delta_{i}(S) b_{j}
$$

where $\delta_{i}(S)$ is the Dirac delta function for a surface $S$ whose boundary is $L$, defined in Appendix IIB. Therefore we find for the displacement (4.8)

$$
\begin{aligned}
& u_{i}^{T}(\mathbf{r})=\frac{1}{8 \pi} \int_{S}\left[R,{ }_{, j k} b_{k} d S_{i}^{\prime}+R,{ }_{j k k} b_{i} d S_{j}^{\prime}\right. \\
& \left.-\frac{1}{1-\nu} R_{, i j k} b_{k} d S_{j}^{\prime}+\frac{\nu}{1-\nu} R_{, i j j} b_{k} d S_{k}^{\prime}\right],
\end{aligned}
$$

where we have used (IIB9). This expression could also have been obtained directly by substituting (2.3) into (II3.30), as was done in ref. [3]. The same result was also obtained by Kroupa [6, 7].

For the discrete dislocation line the dislocation density is given by (II3.23)

$$
\alpha_{i n}(\mathbf{r})=\delta_{i}(L) b_{j},
$$

where $\delta_{i}(L)$ is the Dirac delta function for the closed curve $L$. We therefore find for the elastic distortion (4.13)

$$
\begin{array}{r}
\beta_{i j}(\mathbf{r})=\frac{b_{l}}{8 \pi} \oint_{L}\left[R_{, l n n} \epsilon_{i j k} d L_{k}^{\prime}+R_{, k n n} \epsilon_{i k l} d L_{j}^{\prime}\right. \\
\left.+\left(\frac{R_{, i j k}}{1-\nu}-\delta_{i j} R_{, k n n}\right) \epsilon_{k l m} d L_{m}^{\prime}\right],
\end{array}
$$

where we have used (IIB8). Similarly we find the elastic strain from (4.14) or from (4.23)

$e_{i j}(\mathbf{r})=\frac{b_{l}}{8 \pi} \oint_{L}\left[R, k n n\left(\epsilon_{i k l} d L_{j}^{\prime}\right)_{(i j)}\right.$

$$
\left.+\left(\frac{R, i j k}{1-\nu}-\delta_{i j} R, k n n\right) \epsilon_{k l m} d L_{m}^{\prime}\right]
$$

which agrees with ref. [3]. We find the dilatation from (4.15) or (4.24)

$$
e(\mathbf{r})=-\frac{1-2 \nu}{1-\nu} \frac{b_{l}}{8 \pi} \oint_{L} R_{, k n n} \epsilon_{k l m} d L_{m}^{\prime},
$$

and we find the stress from (4.16)

$$
\begin{aligned}
& \sigma_{i j}(\mathbf{r})=\frac{G b_{l}}{4 \pi} \oint_{L}[R, k n n \\
&\left(\epsilon_{i k l} d L_{j}^{\prime}\right)_{(i j)} \\
&\left.+\frac{1}{1-\nu}\left(R_{, i j k}-\delta_{i j} R_{, k n n}\right) \epsilon_{k l m} d L_{m}^{\prime}\right],
\end{aligned}
$$

which agrees with ref. [3], and has been called the formula of Peach and Koehler. Finally, we find the rotation from $(4.18)$

$$
\omega_{k}(\mathbf{r})=\frac{b_{l}}{8 \pi} \oint_{L}\left[R, l n n d L_{k}^{\prime}-\frac{1}{2} R_{, k n n} d L_{l}^{\prime}\right] .
$$

In this section we have derived explicit closed line integrals for the most useful elastic fields of a discrete dislocation line in an infinite isotropic body. Most of these expressions have already been given before in the literature and proved very valuable for specific calculations and applications of dislocation theory.

\section{Continuous Distribution of Defects}

As in II we shall denote the combination of dislocations and disclinations by the word defects. Similarly we shall refer to the strain and bend-twist as the basic fields. Further discussion of the basic total, elastic, and plastic fields is given in ref. [10].

In this section we give a formal solution to the following problem: The basic plastic fields $e_{i j}^{P}$ and $\kappa_{l j}^{P}$, or the defect densities $\alpha_{i n}$ and $\theta_{i j}$, are given as prescribed functions of space. To find the basic elastic fields $e_{i j}$ and $\kappa_{l j}$.

Again, we solve this problem basically by using the solution for the displacement found in section 3.1. In addition we also derive an expression for the stress. If the basic plastic fields are given then the defect densities are defined by (II4.1-2)

$$
\begin{gathered}
\alpha_{i n} \equiv-\epsilon_{i k l}\left(e_{l n, k}^{P}-\epsilon_{j k n} \kappa_{l j}^{P}\right), \\
\theta_{i j} \equiv-\epsilon_{i k l} \kappa_{l j, k}^{P},
\end{gathered}
$$

which satisfy the continuity conditions (II4.3-4)

$$
\begin{gathered}
\alpha_{i n, i}+\epsilon_{i j n} \theta_{i j}=0, \\
\theta_{i j, i}=0
\end{gathered}
$$


The conditions (5.3-4) can be combined into

$$
\epsilon_{i k l} \alpha_{m l, m k}+\theta_{i l, l}=0 .
$$

The incompatibility (3.17) can also be expressed as (II4.12)

$$
\eta_{i j}=-\left(\epsilon_{j m n} \alpha_{i n, m}+\theta_{i j}\right)_{(i j)} .
$$

Instead of the dislocation density $\alpha_{i n}$ it is sometimes convenient to use the contortion which is still defined as before (I6.5b)

$$
K_{l k}=\frac{1}{2} \delta_{k l} \alpha-\alpha_{k l} .
$$

For the disclination density $\theta_{i j}$ it is useful to have the expression

$$
\begin{aligned}
\theta_{i j}-\delta_{i j} \theta & =-\epsilon_{i k l} \kappa_{l j, k}^{P}+\delta_{i j} \epsilon_{k l m} \kappa_{l m, k}^{P} \\
& =-\epsilon_{i k l}\left(\kappa_{j l, k}^{P}-\kappa_{k l, j}^{P}\right),
\end{aligned}
$$

where the second equality follows from the identity (4.10) with $\alpha$ replaced by $\kappa$. The basic total fields are defined in terms of the total displacement and consist of elastic and plastic parts (II4.14-15)

$$
\begin{gathered}
e_{i j}^{T} \equiv u_{(j, i)}^{T}=e_{i j}+e_{i j}^{P}, \\
\kappa_{l j}^{T} \equiv \omega_{j, l}^{T}=\kappa_{l j}+\kappa_{l j}^{P},
\end{gathered}
$$

where the total rotation is defined by

$$
\omega_{k}^{T} \equiv \frac{1}{2} \epsilon_{i j k} u_{j, i}^{T} .
$$

For completeness we also include the expressions for the characteristic vectors, i.e., the total Burgers vector $B_{n}$ and the Frank vector $\Omega_{j}$ (II $4.5-8$ ):

$$
\begin{aligned}
B_{n} & \equiv-\oint_{\lambda}\left(e_{l n}^{P}-\epsilon_{j m n} \kappa_{i j}^{P} x_{m}\right) d L_{l} \\
& =\int_{\sigma}\left(\alpha_{i n}-\epsilon_{j m n} \theta_{i j} x_{m}\right) d S_{i}, \\
\Omega_{j} & \equiv-\oint_{\lambda} \kappa_{l j}^{P} d L_{l} \\
& =\int_{\sigma} \theta_{i j} d S_{i},
\end{aligned}
$$

where $\lambda$ is a Burgers circuit and $\sigma$ the Burgers surface which spans $\lambda$.

To solve our problem we use the distortion given by (3.10). By some partial integrations and changes in dummy indices we have

$u_{j, i}^{T}(\mathbf{r})=-\frac{1}{8 \pi} \int\left[R_{, l n n} e_{j l, i^{\prime}}^{P}\left(\mathbf{r}^{\prime}\right)+R_{, k n n} e_{k j, i^{\prime}}^{P}\left(\mathbf{r}^{\prime}\right)\right.$ $\left.-\frac{1}{1-\nu} R_{, j k l} e_{k l, i^{\prime}}^{P}\left(\mathbf{r}^{\prime}\right)+\frac{\nu}{1-\nu} R_{, j n n} e_{, i^{\prime}}^{P}\left(\mathbf{r}^{\prime}\right)\right] d V^{\prime}$

By (5.9-11) it is then simple to express the basic elastic fields in terms of the basic plastic fields, which solves the first part of our problem. Next, we wish to find the expressions in terms of the defect densities. First we find from (5.14)

$$
\begin{aligned}
& u_{j, i}^{T}(\mathbf{r})=\frac{1}{8 \pi} \int\left[R_{, l n n}\left\{\epsilon_{i j k}\left(\alpha_{k l}-\kappa_{l k}^{P}\right)-e_{i l, j^{\prime}}^{P}+\epsilon_{i j l \kappa^{P}}\right\}\right. \\
& +R_{, k n n}\left\{\epsilon_{i k l}\left(\alpha_{l j}-\kappa_{j l}^{P}\right)-e_{i j, k^{\prime}}^{P}-\epsilon_{i j k} \kappa^{P}\right\} \\
& -\frac{1}{1-\nu} R_{, j k l}\left\{\epsilon_{i k m}\left(\alpha_{m l}-\kappa_{l m}^{P}\right)-e_{i l, k^{\prime}}^{P}+\epsilon_{i k l} \kappa^{P}\right\} \\
& \left.+\frac{\nu}{1-\nu} R_{, j n n}\left\{\epsilon_{i l m}\left(\alpha_{m l}-\kappa_{l m}^{P}\right)-e_{i l, l^{\prime}}^{P}\right\}\right] d V^{\prime} \\
& =\frac{1}{8 \pi} \int\left[R_{, l n n} \epsilon_{i j k}\left(\alpha_{k l}-\kappa_{l k}^{P}\right)+R_{, k n n} \epsilon_{i k l} \alpha_{i j}\right. \\
& -\frac{1}{1-\nu} R_{, j k l} \epsilon_{i k m} \alpha_{m l}+\frac{\nu}{1-\nu} R_{, j n n} \epsilon_{i l m} \alpha_{m l} \\
& -R_{, n n} \epsilon_{i k l} \kappa_{j l, k^{\prime}}^{P}+\frac{1}{1-\nu} R_{, j} \epsilon_{i k m} \kappa_{l m, k^{\prime}}^{P} \\
& \left.-\frac{\nu}{1-\nu} R_{, j n n} \epsilon_{i l m} \kappa_{l m}^{P}\right] d V^{\prime}+e_{i j}^{P}(\mathbf{r}) \\
& =\frac{1}{8 \pi} \int\left[R_{, l n n} \epsilon_{i j k}\left(\alpha_{k l}-\kappa_{l k}^{P}\right)-R_{, k n n} \epsilon_{i k l} \alpha_{j l}\right. \\
& -R_{, k n n} \epsilon_{k l m} \delta_{i j} \alpha_{m l} \\
& +\frac{1}{1-\nu}\left(R_{, i j k} \epsilon_{k l m}-R_{, j k m} \epsilon_{i k l}\right) \alpha_{m l} \\
& \left.+R_{, n n}\left(\theta_{i j}-\delta_{i j} \theta\right)-\frac{1}{1-\nu} R_{, j l}\left(\theta_{i l}-\delta_{i l} \theta\right)\right] d V^{\prime} \\
& +e_{i j}^{P}(\mathbf{r}) \\
& =\frac{1}{8 \pi} \int\left[R_{, l n n} \epsilon_{i j k}\left(\alpha_{k l}\left(\mathbf{r}^{\prime}\right)-\kappa_{l k}^{P}\left(\mathbf{r}^{\prime}\right)\right)\right. \\
& +R_{, k n n} \epsilon_{i k l} \alpha_{j l}\left(\mathbf{r}^{\prime}\right) \\
& +\left(\frac{R_{, i j k}}{1-\nu}-\delta_{i j} R_{, k n n}\right) \epsilon_{k l m} \alpha_{m l}\left(\mathbf{r}^{\prime}\right) \\
& \left.+R_{, n n} \theta_{i j}\left(\mathbf{r}^{\prime}\right)+\left(\frac{R_{, i j}}{1-\nu}-\delta_{i j} R_{, n n}\right) \theta\left(\mathbf{r}^{\prime}\right)\right] \\
& +e_{i j}^{P}(\mathbf{r}) .
\end{aligned}
$$

Here the first equality follows from (5.1), the second from partial integrations, (3.8), and cancellations, the third from (4.10-11), (5.8), partial integrations and 
cancellations, and the fourth from partial integrations and (5.5).

From (5.15) we then find the elastic strain by (5.9)

$$
\begin{aligned}
& \boldsymbol{e}_{i j}(\mathbf{r})=\frac{1}{8 \pi} \int\left[R_{, k n n}\left(\epsilon_{i k l} \alpha_{j l}\left(\mathbf{r}^{\prime}\right)\right)_{(i j)}\right. \\
& +\left(\frac{R_{, i j k}}{1-\nu}-\delta_{i j} R_{, k n n}\right) \epsilon_{k l m} \alpha_{m l}\left(\mathbf{r}^{\prime}\right)+R_{, n n} \theta_{(i j)}\left(\mathbf{r}^{\prime}\right) \\
& \left.+\left(\frac{R_{, i j}}{1-\nu}-\delta_{i j} R_{, n n}\right) \theta\left(\mathbf{r}^{\prime}\right)\right] d V^{\prime}
\end{aligned}
$$

This is the elastic strain due to the prescribed defect distributions $\alpha_{i n}$ and $\theta_{i j}$. The result could also have been obtained by substituting (2.3) and (2.8) into (II4.29), using (5.4-5). In the latter calculation it will be noted that the terms in (2.8) containing $r_{, q}$ do not contribute to the integral. We see that $e_{i j}$ is a state quantity because it is given in terms of the defect densities. By (5.1-2) we can also express the elastic strain in terms of the basic plastic fields. So (5.16) solves half of the originally posed problem. We note that when the disclination density vanishes, $\theta_{i j}=0$, (5.16) reduces to (4.14), as expected. There is another way to write (5.16), by doing some partial integrations

$$
\begin{aligned}
e_{i j}(\mathbf{r}) & =\frac{1}{8 \pi} \int\left[R, n n\left(\epsilon_{k l m} \alpha_{j l, k^{\prime}}\left(\mathbf{r}^{\prime}\right)+\theta_{i j}\left(\mathbf{r}^{\prime}\right)\right)_{(i j)}\right. \\
+ & \left.\left(\frac{R, i j}{1-\nu}-\delta_{i j} R, n n\right)\left(\epsilon_{k l n} \alpha_{m l, k^{\prime}}\left(\mathbf{r}^{\prime}\right)+\theta\left(\mathbf{r}^{\prime}\right)\right)\right] d V^{\prime},
\end{aligned}
$$

which agrees with (3.22) by (5.6). This then shows an alternative way of deriving (5.16). Next we find the dilatation from (5.16).

$$
\begin{array}{r}
e(\mathbf{r})=-\frac{1-2 \nu}{1-\nu} \frac{1}{8 \pi} \int\left[R_{, k n n} \epsilon_{k l m} \alpha_{m l}\left(\mathbf{r}^{\prime}\right)\right. \\
\left.+R,{ }_{, n n} \theta\left(\mathbf{r}^{\prime}\right)\right] d V^{\prime},
\end{array}
$$

and the stress by (3.2)

$$
\begin{aligned}
\sigma_{i j}(\mathbf{r})= & \frac{G}{4 \pi} \int\left[R,_{k n n}\left(\epsilon_{i k l} \alpha_{j l}\left(\mathbf{r}^{\prime}\right)\right)_{(i j)}\right. \\
& +\frac{1}{1-\nu}\left(R_{, i j k}-\delta_{i j} R,{ }_{, k n n}\right) \epsilon_{k l m} \alpha_{m l}\left(\mathbf{r}^{\prime}\right) \\
& +R_{, n n} \theta_{(i j)}\left(\mathbf{r}^{\prime}\right) \\
& \left.+\frac{1}{1-\nu}\left(R,{ }_{i j}-\delta_{i j} R,{ }_{n n}\right) \theta\left(\mathbf{r}^{\prime}\right)\right] d V^{\prime}
\end{aligned}
$$

To find the elastic bend-twist, we first find the total rotation from (5.11) and (5.15)

$\omega_{k}^{T}(\mathbf{r})=\frac{1}{8 \pi} \int\left[R, l n n\left(\alpha_{k l}-\kappa_{l k}^{P}\right)-\frac{1}{2} R_{, k n n} \alpha\right.$

$$
\begin{aligned}
& \left.+\frac{1}{2} R,{ }_{j n n} \alpha_{j k}+R,{ }_{n n} \epsilon_{i j k} \theta_{i j}\right] d V^{\prime} \\
= & -\frac{1}{8 \pi} \int R_{, l n n}\left[K_{l k}\left(\mathbf{r}^{\prime}\right)+\kappa_{l k}^{P}\left(\mathbf{r}^{\prime}\right)\right] d V^{\prime},
\end{aligned}
$$

where the second equality follows from (5.7), a partial integration, and (5.3). Finally we find the elastic bendtwist

$$
\begin{aligned}
\kappa_{l j}(\mathbf{r})= & -\frac{1}{8 \pi} \int\left[R_{, k \ln n} K_{k j}+R_{, k n n} \kappa_{k j, l^{\prime}}^{P}\right] d V^{\prime}-\kappa_{l j}^{P}(\mathbf{r}) \\
= & -\frac{1}{8 \pi} \int\left[R_{, k l n n} K_{k j}+R_{, k n n}\left(\epsilon_{i k l} \theta_{i j}\right.\right. \\
& \left.\left.-\kappa_{l j, k^{\prime}}^{P}\right)\right] d V^{\prime}-\kappa_{l j}^{P}(\mathbf{r}) \\
= & -\frac{1}{8 \pi} \int\left[R_{, k l n n} K_{k j}\left(\mathbf{r}^{\prime}\right)\right. \\
& \left.+R_{, k n n} \epsilon_{i k l} \theta_{i j}\left(\mathbf{r}^{\prime}\right)\right] d V^{\prime} .
\end{aligned}
$$

Here the first equality follows from (5.10), (5.20), and a partial integration, the second from (5.2), and the third by a partial integration, (3.8), and a cancellation. This is the elastic bend-twist due to prescribed defect distributions $\alpha_{i n}$ and $\theta_{i j}$. The same result can also be obtained by substituting (2.3) into (II4.34), and using (5.3) and (5.5). We see that it is also a state quantity. By (5.1-2) we can also express it in terms of the basic plastic fields. So (5.21) and (5.16) solve the originally posed problem. When the disclination density vanishes, $\theta_{i j}=0,(5.21)$ reduces to the derivative of $(4.18)$, as expected.

In this section we have derived explicit integral expressions for the basic elastic fields and the stress of a. continuous distribution of defects in an infinite isotropic body. We have seen that when the disclination density vanishes, $\theta_{i j}=0$, the results reduce to these for dislocations in section 4.1. Due to the assumption of isotropy the above integrals are given in terms of the relative radius vector $R$ and its derivatives. Hence those are straightforward integrations for any given distribution of defects. For example, in the next section we shall see how they apply to a discrete defect line.

\section{The Discrete Defect Line}

For a discrete defect line along the curve $L$ the basic plastic fields reduce to the special form (II5.14-15)

$$
\begin{aligned}
& e_{l n}^{P}=\beta_{(l n)}^{*}, \\
& \kappa_{l j}^{P}=1 / 2 \epsilon_{j m n} \beta_{m n, l}^{*}+\phi_{l j}^{*},
\end{aligned}
$$

where the defect loop densities are defined by (II5.12-13)

$$
\beta_{l n}^{*}(\mathbf{r}) \equiv-\delta_{l}(S)\left\{b_{n}+\epsilon_{j m n} \Omega_{j}\left(x_{m}-x_{m}^{0}\right)\right\},
$$




$$
\phi_{l j}^{*}(\mathbf{r}) \equiv-\delta_{l}(S) \Omega_{j} .
$$

Here $b_{n}$ and $\Omega_{j}$ are the characteristic vectors, i.e., the dislocation Burgers vector and the Frank vector, $x_{m}^{0}$ is a point thru which the axis of the disclination passes, and $\delta_{l}(S)$ is the Dirac delta function for a surface $S$ whose boundary is $L$. The defect densities are given by (II5.19-20)

$$
\begin{aligned}
\alpha_{i n}(\mathbf{r}) & =\delta_{i}(L)\left\{b_{n}+\epsilon_{j m n} \Omega_{j}\left(x_{m}-x_{m}^{0}\right)\right\}, \\
\theta_{i j}(\mathbf{r}) & =\delta_{i}(L) \Omega_{j},
\end{aligned}
$$

where $\delta_{i}(L)$ is the Dirac delta function for the closed curve $L$. The contortion is by (5.7) and (6.5)

$$
\begin{aligned}
K_{k j}(\dot{\mathbf{r}})=1 / 2 \delta_{j k} \delta_{i}(L)\left\{b_{i}+\epsilon_{i p q} \Omega_{p}\left(x_{q}-x_{q}^{0}\right)\right\} \\
-\delta_{j}(L)\left\{b_{k}+\epsilon_{k p q} \Omega_{p}\left(x_{q}-x_{q}^{0}\right)\right\} .
\end{aligned}
$$

The total Burgers vector is (II5.17)

$$
B_{n}=b_{n}-\epsilon_{j m n} \Omega_{j} x_{m}^{0},
$$

and the displacement jump across the surface $S$ is (II5.1, II5.5)

$$
\left[u_{n}\right]=B_{n}+\epsilon_{j m n} \Omega_{j} x_{m}=b_{n}+\epsilon_{j m n} \Omega_{j}\left(x_{m}-x_{m}^{0}\right) .
$$

We now find the total displacement from (3.9), (6.1), and (6.3)

$$
\begin{aligned}
u_{i}^{T}(\mathbf{r})=\frac{1}{8 \pi} \int_{S}\left\{b_{k}+\epsilon_{k m n} \Omega_{m}\left(x_{n}^{\prime}-x_{n}^{0}\right)\right\} \\
{\left[R,{ }_{, j k} d S_{i}^{\prime}+R_{, j l l} \delta_{i k} d S_{j}^{\prime}\right.} \\
\left.-\frac{1}{1-\nu} R_{, i j k} d S_{j}^{\prime}+\frac{\nu}{1-\nu} R_{, i j j} d S_{k}^{\prime}\right],
\end{aligned}
$$

where we have used (IIB9). When the disclination vanishes, $\Omega_{m}=0$, this equation reduces to (4.21). We find the elastic strain from (5.16) and (6.5-6).

$$
\begin{gathered}
e_{i j}(\mathbf{r})=\frac{1}{8 \pi} \oint_{L}\left[\left\{b_{l}+\epsilon_{l p q} \Omega_{p}\left(x_{q}^{\prime}-x_{q}^{0}\right)\right\}\right. \\
\left\{R_{, k n n}\left(\epsilon_{i k l} d L_{j}^{\prime}\right)_{(i j)}\right. \\
\left.+\left(\frac{R_{, i j k}}{1-\nu}-\delta_{i j} R_{, k n n}\right) \epsilon_{k l m} d L_{m}^{\prime}\right\} \\
\left.+R_{, n n}\left(\Omega_{j} d L_{i}^{\prime}\right)_{(i j)}+\left(\frac{R_{, i j}}{1-\nu}-\delta_{i j} R_{, n n}\right) \Omega_{m} d L_{m}^{\prime}\right],
\end{gathered}
$$

where we have used (IIB8). Again, for $\Omega_{m}=0$, this reduces to (4.24).
The dilatation becomes

$$
\begin{array}{r}
e(\mathbf{r})=-\frac{1-2 \nu}{1-\nu} \frac{1}{8 \pi} \oint_{L}\left[\left\{b_{l}+\epsilon_{l p q} \Omega_{p}\left(x_{q}^{\prime}-x_{q}^{0}\right)\right\}\right. \\
\left.R_{, k n n} \epsilon_{k l m}+R_{, n n} \Omega_{m}\right] d S_{m}^{\prime},
\end{array}
$$

and the stress becomes

$$
\begin{aligned}
& \sigma_{i j}(\mathbf{r})=\frac{1}{8 \pi} \oint_{L}\left[\left\{b_{l}+\epsilon_{l p q} \Omega_{p}\left(x_{q}^{\prime}-x_{q}^{0}\right)\right\}\right. \\
& \left\{R_{, k n n}\left(\epsilon_{i k l} d L_{j}^{\prime}\right)_{(i j)}+\frac{1}{1-\nu}\left(R_{, i j k}-\delta_{i j} R_{, k n n}\right) \epsilon_{k l m} d L_{m}^{\prime}\right\} \\
& \left.\quad+R_{, n n}\left(\Omega_{j} d L_{i}^{\prime}\right)_{(i j)}+\frac{1}{1-\nu}\left(R_{, i j}-\delta_{i j} R_{, n n}\right) \Omega_{m} d L_{m}^{\prime}\right] .
\end{aligned}
$$

Finally we find the elastic bend-twist from (5.21) and $(6.6-7)$

$$
\begin{aligned}
& \kappa_{l j}(\mathbf{r})=\frac{1}{8 \pi} \oint_{L}\left[\left\{b_{k}+\epsilon_{k p q} \Omega_{p}\left(x_{q}^{\prime}-x_{q}^{0}\right)\right\}\right. \\
&\left.\left\{R_{, k l n n} d L_{j}^{\prime}-\frac{1}{2} R_{, j l n n} d L_{k}^{\prime}\right\}-R_{, k n n} \epsilon_{i k l} \Omega_{j} d L_{i}^{\prime}\right] .
\end{aligned}
$$

In this section we have derived explicit closed line integrals for the basic elastic fields and the stress of a discrete defect line in an infinite isotropic body. We have seen that when the disclination vanishes, $\Omega_{m}=0$, the results reduce to those for a discrete dislocation line in section 4.2. All the above integrals are given in terms of radius vectors. Hence they should be straightforward for given geometries of the discrete defect lines. As in the case of dislocation lines this should make the above expressions useful for the practical application of disclinations.

\section{Summary}

We have specialized previous results derived for anisotropic disclination theory to the special case of isotropy. We have also derived the isotropic theory independently and showed that anisotropic theory reduces to it.

We have given a short review of incompatible theory which serves as the basis of departure for all subsequent applications to defects. We have also reviewed dislocation theory which forms a basis of comparison for defect theory.

For a continuous distribution of defects we have derived the expressions for the state quantities: the basic elastic fields and the stress. These expressions are given in terms of the defect densities, the disclination and the dislocation density, which in turn can be expressed in terms of the basic plastic fields. Next, 
we specialized these results to a discrete defect loop. These expressions are given in terms of the characteristic vectors, the total Burgers vector and the Frank vector, as line integrals along the defect line. We have shown explicitly that these results reduce to those for dislocation theory when the disclinations vanish.

The results for the discrete defect line are in a useful form for application to special geometries. In future publications we shall apply them to straight lines [8] and loops [9].

\section{References}

[1] deWit, R., in Fundamental aspects of dislocation theory, Nat. Bur. Stand. (U.S.) Spec. Publ. 317, Vols. 1 and 2 (1970), p. 651. In this paper referred to as I.
[2] deWit, R., J. Res. Nat. Bur. Stand. (U.S.) 77A, (Phys. and Chem.), No. 1, 49-100 (Jan.-Feb. 1973). In this paper referred to as II.

[3] deWit, R., Sol. State Phys. 10,269 (1960).

[4] Eshelby, J. D., Progr. Solid Mech. II, 89 (1961).

[5] Eshelby, J. D., Sol. State Phys. 3, 79 (1956).

[6] Kroupa, F., Czech. J. Phys. B12, 191 (1962).

[7] Kroupa, F., in Theory of Crystal Defects, Ed., B. Gruber (Academic Press, N. Y., 1966), p. 275.

[8] deWit, R., Theory of Disclinations IV. Straight Disclinations (to be published).

[9] deWit, R., Theory of Disclinations V. Disclination Loops (to be published).

[10] deWit, R., Continuous Distribution of Disclination Loops, submitted to phys. stat. sol.

(Paper 77A3-775) 